\title{
A Influência do Marketing Verde no Consumo Sustentável Uma Survey no Rio Grande do Norte
}

http://dx.doi.org/10.21527/2237-6453.2020.51.268-280

Recebido em: 28/6/2019

Aceito em: 5/12/2019

\author{
Eliana Andréa Severo, ${ }^{1}$ Ádina Swanne Francelino Barbosa, ${ }^{2}$ \\ Suzyanne Maria Mota, ${ }^{3}$ Marcelo de Brito Oliveira ${ }^{4}$
}

\begin{abstract}
RESUMO
A problemática ambiental impacta o meio ambiente e torna-se um fator primordial na decisão de compra dos consumidores. O objetivo deste estudo consiste em analisar a influência do marketing verde no consumo sustentável perante a percepção de 820 consumidores do Rio Grande do Norte (RN). A metodologia utilizada foi uma pesquisa quantitativa e descritiva, por meio da análise fatorial confirmatória e da regressão linear múltipla. Os resultados evidenciam que o marketing verde apresenta uma intensidade moderada e influencia em $43,7 \%$ o consumo sustentável no RN. Estes achados mostram que o marketing verde contribui para comportamentos sustentáveis, pois evidencia-se que os consumidores percebem a problemática ambiental, bem como influencia o poder de compra dos consumidores, assim como pode torná-los futuros consumidores verdes.
\end{abstract}

Palavras-chave: Marketing verde. Consumo sustentável. Consumidores.

\section{THE INFLUENCE OF GREEN MARKETING ON SUSTAINABLE CONSUMPTION: A SURVEY IN RIO GRANDE DO NORTE}

\section{ABSTRACT}

The environmental problem impacts the environment, and is a key factor in the decision to purchase consumers. The objective of this study is to analyze the influence of green marketing on sustainable consumption to the perception of 820 consumers in Rio Grande do Norte (RN). The methodology used was a quantitative and descriptive research, through confirmatory factorial analysis and multiple linear regression. The results show that the green marketing has a moderate intensity and influences $43.7 \%$ sustainable consumption in the RN. These findings indicate that green marketing contributes to sustainable behaviors because it is evident that consumers perceive the environmental problem as well as influences the purchasing power of consumers, as well as making them future green consumers.

Keywords: Green marketing. Sustainable consumption. Consumers.

\footnotetext{
${ }^{1}$ Doutora em Administração pela Pontifícia Universidade Católica do Rio Grande do Sul (PUCRS). Professora do Mestrado Profissional em Gestão Empresarial (MPGE) do Centro Universitário UniFBV/Wyden em Boa Viagem, Recife - PE. elianasevero2@hotmail.com

${ }^{2}$ Graduada em Administração pela Universidade Potiguar (UNP). adina.swanne@hotmail.com

${ }^{3}$ Graduada em Administração pela Universidade Potiguar (UNP). anne_mota-04@hotmail.com

${ }^{4}$ Graduado em Administração pela Universidade Potiguar (UNP). marcelo-brito07@hotmail.com
} 
Atualmente a sociedade está inserida em um mundo capitalista, no qual a grande produção industrial e o consumo exacerbado estão impactando o meio ambiente e a qualidade de vida da sociedade. As consequências desses atos levam ao desmatamento, poluição, destinação inadequada de resíduos, entre outros males; entretanto as organizações vêm sendo pressionadas e motivadas pela legislação ambiental e a sociedade, que estão na busca por produtos verdes. Neste cenário, torna-se notável como o marketing proveniente dessas empresas influencia na hora da decisão de compra de seus consumidores.

O marketing é capaz de influenciar os consumidores na hora da compra bem como nas suas mudanças de hábitos de acordo com as necessidades ocorridas pelo mercado, buscando, assim, satisfazê-las de modo eficaz. É necessário, porém, que as empresas possam atender o nicho de clientes fiéis à marca sustentável, e, assim, conquistar uma melhor performance organizacional. Termos como eco, ecológico, menos poluente e sustentável, estão ganhando espaço nas embalagens para vender ao consumidor a imagem de empresa ambientalmente correta e do produto verde (CHEN; CHANG, 2013; DELLARMELIN et al., 2018; CHANG et al., 2019). Neste sentido, surge o marketing verde com o propósito de transmitir ao consumidor as práticas de responsabilidade social corporativa da empresa (RSC), as práticas ambientais e o investimento da empresa junto as questões ambientais para a preservação do meio ambiente (OTTMAN; PARO, 1994; NYILASY, GANGADHARBATLA; PALADINO, 2014; PAPADAS; AVLONITIS; CARRIGAN, 2017).

Estes termos, contudo, podem gerar no consumidor a confiança no produto verde e no consumo verde ou a confusão sobre o consumo verde. Por este motivo, passou a ser discutido o greenwashing, ou a maquiagem verde, feita pelas empresas em suas ações de marketing (DAHL, 2010; TIWARI et al., 2011; CHEN; CHANG, 2013; NYILASY; GANGADHARBATLA; PALADINO, 2014) e, assim, discute-se o questionamento de que o greenwashing pode influenciar na percepção do consumidor para o consumo verde. As organizações devem, entretanto, apresentar eticamente seus produtos verdes para a sociedade, para atestar o que de fato são os produtos ambientalmente corretos (ZHANG et al., 2018).

O consumo verde é dominado por cogitações que consideram que os consumidores alcancem conhecimento suficiente para ganhar a necessária consciência ambiental, traduzindo-a em atitudes e comportamentos ambientalmente benignos. A realidade, no entanto, traz fatores determinantes para a mudança na prática dos consumidores, tal como valor agregado a esses produtos. Paavola (2001) alerta para o fato de que a estratégia de consumo verde enfatiza produtos elitizados para uma parcela da sociedade por seu valor diferenciado. Existe, contudo, uma crescente conscientização dos consumidores em relação aos problemas ambientais; assim, o marketing verde está tornando-se uma importante estratégia para as empresas obterem uma vantagem competitiva.

Perante o exposto, este estudo tem como objetivo analisar a influência do marketing verde na adoção do consumo sustentável por meio da percepção de 820 consumidores do Rio Grande do Norte (RN). Consoante isso, este estudo visa a responder a seguinte questão de pesquisa: Qual a influência do marketing verde no consumo sustentável? Por conseguinte, a pesquisa pretende contribuir para a conscientização do 
consumo de produtos verdes, tendo como base analisar a influência na esfera do consumo sustentável como não prejudicial ao meio ambiente, indagando, assim, sobre as dificuldades em lidar com problemas nesta dimensão.

\section{REFERENCIAL TEÓRICO}

\section{Marketing Verde}

Segundo Ottman (1993), Beylot et al. (2019) e Ryan, Danylchuk e Jordaan (2019), em meados da década de 70 os impactos ambientais, bem como seu poder de transformação e influência, são percebidos mais fortemente. Conforme Ottman (1993), a exigência de conscientização nesse aspecto passa a se estender não somente às pessoas físicas, mas, principalmente, às pessoas jurídicas, que passaram a perceber a adoção dos produtos verdes como um diferencial competitivo.

De acordo com Ottman (1993) e Chang et al. (2019), com o passar dos anos os valores ambientais mudaram de um interesse para outro, ou seja, a opção cresceu para o topo da agenda da nação, e, com isso, as preocupações ambientais vieram à tona, adequando-se em meados de 1990, aderindo, então, a respostas a inúmeras iniciativas legislativas destinadas a corrigir os problemas ocorridos no dia a dia dos consumidores.

Para Straughan e Roberts (1999) e Guillen-Royo (2019), a partir do surgimento das questões de impacto ambiental buscam-se indicadores para poder explicar a viabilidade de as pessoas terem um comportamento ecologicamente consciente. Neste cenário, não basta apenas ser adepto do marketing verde; o produto final deve ser semelhante ou superior aos outros para realmente agradar os consumidores que, muitas vezes, ainda visualizam apenas o custo-benefício individual, sem reparar no custo-benefício em longo prazo para o meio ambiente (FRANCO, 2016; XU et al., 2018).

Alguns estudos expõem o impacto das emoções sobre a satisfação (LADHARI, 2007). Pesquisas ainda mostram que a resposta emocional corresponde, primorosamente, a diferentes percepções de satisfação, confiança e compromisso (SCHOEFER; DIAMANTOPOULOS, 2009), de forma que as emoções geram impacto positivo no consumo e no boca a boca positivo (DAS; VARSHNEYA, 2017).

Diante disso, os consumidores estão sujeitos a estímulos de marketing que são vistos como indutores de emoções sob a forma de propagandas, embalagens de produtos, posicionamento de marcas, eventos, entre outros (LEE; ANDRADE; PALMER, 2013; CHEN; MATHUR; MAHESWARAN, 2014; HOFFMANN et al., 2019).

Nas avaliações de segmento de mercado, propostas por Engel, Blackwell e Miniard (2000) e por Straughan e Roberts (1999), foi concluído ser indiscutível a existência de um grupo de consumidores verdes, e que as variáveis psicográficas são mais apropriadas que as demográficas para essa identificação.

Neste cenário, fica evidente que a prática da questão ambiental é um fator primordial na decisão de compra dos consumidores, e isso tem gerado uma grande expansão de produtos verdes em diversos países, acompanhado de várias oportunidades de negócios geradas com o uso do marketing voltado ao consumidor desses produtos (DIAMANTOPOULOS et al., 2003). 
O marketing verde é um conjunto de políticas e estratégias de comunicação que visam à obtenção da vantagem comparativa de diferenciação para os produtos ou serviços que oferece a organização em relação às marcas concorrentes, consolidando seu posicionamento competitivo (DIAS, 2008; DANGELICO; VOCALELLI, 2017). A prática do marketing verde significa divulgar uma preocupação ambiental com as fontes de entrada e saída na fabricação de um produto ou na prestação de um serviço (WALKER; HANSON, 1998).

De acordo com Kotler e Keller (2012), o marketing socialmente responsável deve partir de três frentes: 1 ) comportamento legal, conectado ao cumprimento das leis; 2 ) comportamento ético, propõe uma elaboração de um código ético e divulgar essas premissas para todos conhecerem; e, 3) comportamento de responsabilidade social e compromisso com o social e ambiental.

\section{Consumo Sustentável}

O consumo sustentável é a forma de consumir bens e serviços que respeitam o meio ambiente, não o degradando e preservando-o para as gerações futuras (SEVERO; GUIMARÃES; DORION, 2018). Neste sentido, busca-se a transformação para o consumo sustentável, dependendo das mudanças fundamentais na visão mundial, rompendo padrões de dimensões econômica, social, ambiental, ética, focados em direção a um novo sistema ecológico (LIM, 2017). Mediante isso, é necessário que a cultura e os valores pessoais estejam alinhados com o comportamento sustentável (SHARMA; JHA, 2017).

De acordo com Portilho (2005), a atividade de consumo e o próprio papel do consumidor podem oferecer importantes mudanças de constituição de sujeitos sociais ativos e de retorno benéfico ao cidadão. Os consumidores verdes podem ser considerados aqueles que se preocupam com e julgam - durante o seu processo de compra - fatores como: recursos despendidos para a fabricação do produto, descarte posterior do produto, possibilidade de serem reciclados, entre outros (ZINKHAN; CARLSON, 1995).

Em virtude do consumidor verde o marketing verde existe, bem como é uma preocupação real das empresas (D'SOUZA; TAGHIAN; LAMB, 2006; ZHANG et al., 2018). Prahalad e Hart (2000) ressaltam, contudo, que, como uma vasta classe em crescimento de consumo, faz-se necessário reconhecer quais são as principais características desse mercado, buscando destacar os pontos de relevância e, com isso, reconhecer as ações que podem ser desenvolvidas para que haja o consumo sustentável.

Para Alves (2016) e Dong et al. (2018), o consumidor não compra um produto somente porque ele é verde, mas porque tem funcionalidade, desempenho, design, preço adequado e todos os demais atributos que ele espera. Neste contexto, para promover a compra de produtos sustentáveis e transmitir o conhecimento em relação às marcas sustentáveis, torna-se necessário ampliar a educação e a conscientização que motivam um comportamento de consumo verde e podem levar à compra consciente, dado que os consumidores jovens tendem a ter um consumo mais sustentável se os mesmos compreendem o conceito de consumo sustentável (GENG; LIU; ZHU, 2017).

Conforme Gomes (2006), a consciência de consumo é questão principal para o desenvolvimento da responsabilidade socioambiental do cidadão. Assim, surge o consumidor verde, indivíduo capaz de alterar seu consumo em prol da defesa do meio ambiente, utilizando-se de medidas preventivas (SHETH; MITTAL; NEWMAN, 2001). 
O consumo sustentável envolve uma escolha cuidadosa do que comprar, prestando atenção se o produto é social e ambientalmente responsável, se sua produção envolve condições dignas aos trabalhadores, se reduz o gasto de recursos naturais, se envolve reciclagem ou reutilização das embalagens e se é realmente uma aquisição necessária (FRANCO, 2016; DELLARMELIN et al., 2018). Em suma, é um consumo que pretende não esgotar os recursos naturais, tendo em vista o futuro do planeta e das próximas gerações. Sob essa perspectiva, diminui-se o gasto desenfreado de recursos naturais, evita-se o desperdício e incentiva-se o consumo que preze pela qualidade de vida (FRANCO, 2016).

Franco (2016) e Dhandra (2019) ressaltam que o consumo sustentável pode levar tempo para ser efetivado, mas se torna mais fácil de ser um hábito quando passar a ser um valor da sociedade e esse valor tornar-se natural. Ainda conforme Dhandra (2019), a intenção de compra verde, a consciência ambiental e o materialismo mediaram significativamente a relação entre atenção plena e satisfação com a vida; isso indica como a atenção plena gera um senso de cuidado com a natureza e a sociedade.

\section{METODOLOGIA}

A metodologia utilizada neste estudo é a pesquisa quantitativa e descritiva por meio de uma Survey, que analisa as relações entre marketing verde e consumo sustentável perante a percepção de 820 consumidores do RN. Segundo Marconi e Lakatos (2017) e Hair Jr. et al. (2013), a pesquisa quantitativa é conhecida pelo uso da quantificação, na coleta e também no tratamento das informações geradas pelo uso de técnicas estatísticas, evitando distorções de análises e de interpretação e também garantindo uma margem de segurança maior quanto às interferências.

Na pesquisa quantitativa, o método Survey, conforme Malhotra (2012), é o principal método utilizado em pesquisas descritivas. Coerentemente, assume-se como um levantamento do tipo Survey um universo de centenas ou milhares de elementos (HAIR JR. et al., 2013; MALHOTRA, 2012).

Para Malhotra (2012), a pesquisa descritiva é uma pesquisa conclusiva, que tem como seu principal objetivo a descrição de algo. A maior parte da pesquisa de mercado comercial é descritiva em sua natureza por ser muito útil quando perguntas da pesquisa são relativas à descrição de um fenômeno de mercado, como a frequência de compra, a identificação de relacionamentos ou a elaboração de previsões.

Neste cenário, Gil (2006) e Marconi e Lakatos (2017) afirmam que as pesquisas descritivas têm como objetivo primordial a descrição das características de determinada população ou fenômeno ou, então, o estabelecimento de relações entre variáveis. São inúmeros os estudos que podem ser classificados sob este título, e uma de suas características mais significativas está na utilização de técnicas padronizadas de coleta de dados, tais como o questionário e a observação sistemática.

De acordo com Malhotra (2012), os estudos descritivos são usados para retratar variáveis do mercado, que descrevem o tamanho do mercado e medem a frequência de comportamentos, como o de compras. Entre os principais tipos de estudos descritivos estão os de vendas focados interna e externamente, os de percepção e comportamento de consumidores e os de características do mercado. 
No que se refere à população investigada, o Estado do RN é constituído por 3.479.010 habitantes (IBGE, 2018). Por conseguinte, a amostra é caracterizada como não probabilística, por conveniência (HAIR JR. et al. 2013), constituída por 820 respondentes. Assim, a coleta de dados foi realizada sob o aspecto de bola de neve, ou seja, pelos contatos e redes sociais dos pesquisadores, quando houve uma maior abrangência no Estado do RN no período de setembro de 2018 a fevereiro de 2019. Para a coleta de dados elaborou-se um questionário com 16 questões, em que 6 perguntas estão relacionadas à questões demográfica, gênero, idade, escolaridade e renda familiar, e 10 questões (Figura 1) apresentam afirmações por meio de uma escala Likert de 5 pontos, que variam de discordo totalmente a concordo totalmente, sendo: i) 1 = Discordo totalmente; ii) 2 = Discordo parcialmente; iii) 3 = Nem discordo, nem concordo; iv) 4 = Concordo parcialmente; e, v) 5 = Concordo totalmente. Byrne (2010) e Guimarães et al. (2016) observam que a escala do tipo Likert, com cinco pontos, não tem originalmente uma distribuição contínua, porém ela cumpre o requisito de continuidade quando utilizada de forma somada e com o uso das médias das respostas.

Neste contexto, o primeiro Construto apresenta a importância de se adotar um Consumo Sustentável e o impacto desse comportamento, no qual as questões foram adaptadas dos estudos de Oliveira, Gomes e Correia (2018) e Lira (2018); já no segundo Construto é avaliada a influência do Marketing Verde na hora da compra, sendo as questões adaptadas dos estudos de Braga Junior (2016) e Severo et al. (2017). Destaca-se que antes da aplicação o questionário passou pela validação de dois doutores expert na área temática estudada. Consoante isso, realizou-se um pré-teste com 21 respondentes para o entendimento das questões.

Figura 1 - Construtos da Pesquisa

\begin{tabular}{|c|c|c|}
\hline \multicolumn{3}{|c|}{ Construto - Consumo Sustentável } \\
\hline Sigla & Questões & Autores \\
\hline CS1 & Considero importante o consumo sustentável no dia a dia. & \multirow{5}{*}{$\begin{array}{l}\text { Adaptado dos } \\
\text { estudos de Oli- } \\
\text { veira, Gomes e } \\
\text { Correia (2018) e } \\
\text { Lira (2018) }\end{array}$} \\
\hline CS2 & $\begin{array}{l}\text { O fator do produto ser ecologicamente sustentável me influencia na hora } \\
\text { da compra. }\end{array}$ & \\
\hline CS3 & $\begin{array}{l}\text { Acredito que na compra do produto verde é possível obter impacto posi- } \\
\text { tivo no meio ambiente. }\end{array}$ & \\
\hline CS4 & $\begin{array}{l}\text { Considero que o valor dos produtos sustentáveis é acessível para o con- } \\
\text { sumo cotidiano. }\end{array}$ & \\
\hline CS5 & $\begin{array}{l}\text { Estou consciente que o consumo de produtos sustentáveis (verdes) é } \\
\text { importante para o meio ambiente e a manutenção das gerações futuras. }\end{array}$ & \\
\hline \multicolumn{3}{|c|}{ Construto - Marketing Verde } \\
\hline Sigla & Questões & Autores \\
\hline MKT1 & $\begin{array}{l}\text { A propaganda tem uma grande influência na escolha dos produtos sus- } \\
\text { tentáveis. }\end{array}$ & \multirow{5}{*}{$\begin{array}{l}\text { Adaptado dos } \\
\text { estudos de Braga } \\
\text { Junior (2016) e } \\
\text { Severo et al. } \\
\text { (2017) }\end{array}$} \\
\hline MKT2 & $\begin{array}{l}\text { A propaganda sobre produtos sustentáveis me levou a realizar uma com- } \\
\text { pra de produto verde. }\end{array}$ & \\
\hline MKT3 & $\begin{array}{l}\text { Se as lojas, onde habitualmente compro, oferecerem produtos verdes eu } \\
\text { substituiria a compra de produtos convencionais por produtos verdes. }\end{array}$ & \\
\hline MKT4 & $\begin{array}{l}\text { Considero importante uma melhor divulgação sobre o descarte correto } \\
\text { de resíduos eletrônicos (pilhas, baterias, celulares, TV) }\end{array}$ & \\
\hline MKT5 & $\begin{array}{l}\text { Considero importante a divulgação do consumo consciente dos produtos } \\
\text { verdes. }\end{array}$ & \\
\hline
\end{tabular}

Fonte: Elaborada pelos autores (2018). 
Para a análise dos dados, primeiramente utilizou-se a técnica de estatística descritiva. Para Hair Jr. et al. (2013) e Malhotra (2012), a estatística descritiva é utilizada para transcrever os dados de uma determinada amostra utilizando os dois tipos de medidas, como de tendência central e de dispersão. Neste cenário utilizou-se a média e o percentual para a análise dos resultados.

Posteriormente, empregou-se a análise multivariada de dados, por meio da análise fatorial confirmatória (AFC) e a regressão linear múltipla. Conforme Malhotra (2012), a análise fatorial procura encontrar fatores em um grupo de variáveis explicativas para um determinado fenômeno, representando uma classe de processos utilizados para a sumarização de dados originalmente contida em um grupo de variáveis em um conjunto de fatores (HAIR JR. et al., 2013). Já a regressão linear múltipla usa medidas que buscam explorar a relação entre as variáveis estudadas (HAIR JR. et al., 2013).

Para o tratamento e análise dos dados, por meio da análise fatorial confirmatória e regressão linear múltipla, utilizou-se uma planilha do software Microsoft Excel ${ }^{\circledR}$ (2007), bem como o software SPSS ${ }^{\circledR}$ Versão 21 para Windows. Neste contexto, também se utilizou os testes de Kaiser-Meyer-Olkin (KMO) de adequação das amostras e o Teste de Esfericidade de Bartlett para verificar a normalidade e consistência interna dos dados.

\section{ANÁLISE E DISCUSSÃO DOS RESULTADOS}

Para a depuração dos dados, buscou-se identificar outliers univariados e multivariados, análise de simetria de Pearson com valores próximos a Zero (HAIR JR. et al., 2013) e a análise da Curtose, com valores inferiores a 5 (MARDIA, 1971; HAIR JR. et al., 2013), bem como foram eliminados os formulários em que o respondente concentrou as respostas em uma única alternativa da escala Likert de cinco pontos. Observa-se que o formulário eletrônico não permitia não respostas (missing). Inicialmente foram coletados 843 questionários, dos quais eliminou-se 23 formulários (outliers), o que resultou em 820 casos válidos.

O processamento dos dados revelou que na amostra de 820 respondentes $60,7 \%$ eram do gênero feminino, $38,2 \%$ do masculino e $1,1 \%$ eram de outros gêneros. A maior parte dos respondentes, $52,4 \%$, foi composta, principalmente, por jovens da faixa etária de 18 a 28 anos. Os demais ficaram divididos em: 23,2\% de 29 a 39 anos; $15,5 \%$ de 40 a 50 anos; e, 8,9\% acima de 51 anos.

Com relação ao estado civil, a amostra apresenta $50,7 \%$ dos entrevistados solteiros, 37,4\% casados, 8,3\% com união estável, 2,2\% divorciados e, 1,3\% viúvos. Quanto à escolaridade, grande parte dos respondentes possui nível superior incompleto, 40,2\%, 26,8\% possuem Ensino Superior completo, 10,7\% possuem Pós-Graduação completa, 12,4\% possuem Ensino Médio completo, 5,1\% possuem Pós-Graduação incompleta, 2,9\% possuem Ensino Médio incompleto, 1,2\% possuem Ensino Fundamental incompleto e $0,5 \%$ possuem Ensino Fundamental completo.

Em relação à renda familiar, grande parte dos entrevistados, 51,2\%, recebem acima de três salários mínimos, enquanto $24,6 \%$ até 3 salários mínimos; $21,1 \%$ recebem até 2 salários mínimos e $3 \%$ até 1 salário mínimo. 


\section{Análise Fatorial Confirmatória}

Antes do processo de validação das variáveis observáveis foram realizados os testes de Kaiser-Meyer-Olkin (KMO) de adequação das amostras e o Teste de Esfericidade de Bartlett, os quais indicam se as variáveis encontram-se correlacionadas, possibilitando a utilização da técnica de análise fatorial (HAIR JR. et al., 2013). A Tabela 1 destaca que o KMO apresenta um valor acima de $0,7(0,776)$, o que indica que a análise fatorial é uma técnica adequada para análise dos dados (MALHOTRA, 2012; HAIR JR. et al., 2013), bem como o Teste de Esfericidade de Barlett (Tabela 1 ) foi significativo $(0,00)$, indicando que existe correlação entre as variáveis, sendo adequado ao uso da análise fatorial.

Tabela 1 - KMO e Esfericidade de Barlett

\begin{tabular}{|l|l|c|}
\hline \multicolumn{2}{|c|}{ Teste } & Valor Encontrado \\
\hline KMO & 0,776 \\
\hline \multirow{2}{*}{ Teste de Esfericidade de Bartlett } & Qui-quadrado & 1531,02925394271 \\
\cline { 2 - 3 } & Significância & $0,000^{*}$ \\
\hline
\end{tabular}

$*<0,001$

Fonte: Dados provenientes da pesquisa quantitativa (2018).

Neste sentido, realizou-se a análise fatorial confirmatória intrablocos da pesquisa, que abrange 2 Blocos: i) Bloco 1 - Construto de Consumo Sustentável (CS); e ii) Bloco 2 - Construto de Marketing Verde (MKT). No que se refere à análise fatorial confirmatória para o Bloco 1 - CS, este apresenta valores aceitáveis à validação da escala (Tabela 2), pois a comunalidade é aceitável, posto que quatro variáveis observáveis apresentam valores superiores a 0,5 , assim como as cargas fatoriais com valores acima de 0,5 , segundo preconizam Hair Jr. et al. (2013). Apenas a variável CS4, entretanto, apresenta uma baixa comunalidade $(0,328)$, porém esta questão mostra uma carga fatorial aceitável $(0,572)$. Consoante isso, Lee e Hooley (2005) ressaltam que uma variável de comunalidade baixa não deve ser automaticamente excluída da análise, pois o pesquisador pode não apenas analisar a relação com o objeto de estudo, mas também resumi-la a um nível ideal para todas as pesquisas. Optou-se, assim, por manter esta questão no construto, pois ela é essencial para verificar a importância do consumo sustentável.

Tabela 2 - Análise fatorial confirmatória para o Consumo Sustentável

\begin{tabular}{|c|c|c|c|}
\hline Bloco & Variáveis observáveis & Comunalidade & $\begin{array}{l}\text { Cargas } \\
\text { Fatoriais }\end{array}$ \\
\hline \multirow{5}{*}{$\begin{array}{l}\text { Consumo } \\
\text { Sustentável } \\
\text { (CS) }\end{array}$} & $\begin{array}{l}\text { CS1 - Considero importante o consumo sustentável } \\
\text { no dia a dia. }\end{array}$ & 0,666 & 0,816 \\
\hline & $\begin{array}{l}\text { CS2 - O fator do produto ser ecologicamente sus- } \\
\text { tentável me influencia na hora da compra. }\end{array}$ & 0,584 & 0,764 \\
\hline & $\begin{array}{l}\text { CS3 - Acredito que na compra do produto verde é } \\
\text { possível obter impacto positivo no meio ambiente. }\end{array}$ & 0,618 & 0,786 \\
\hline & $\begin{array}{l}\text { CS4 - Considero que o valor dos produtos sustentá- } \\
\text { veis é acessível para o consumo cotidiano. }\end{array}$ & 0,328 & 0,572 \\
\hline & $\begin{array}{l}\text { CS5 - Estou consciente que o consumo de produtos } \\
\text { sustentáveis (verdes) é importante para o meio am- } \\
\text { biente e a manutenção das gerações futuras. }\end{array}$ & 0,635 & 0,797 \\
\hline
\end{tabular}

Fonte: Dados da pesquisa (2018). 
Em relação às cargas fatoriais no Bloco 1, a variável observável CS1 (Considero importante o consumo sustentável no dia a dia) apresentou o maior índice $(0,816)$, demonstrando que está contribuindo significativamente para a composição do Bloco 1 , corroborando os pressupostos de Dellarmelin et al. (2018) e Dong et al. (2018), pois a preocupação ambiental está ganhando espaço nas embalagens ao transmitir ao consumidor a imagem de empresa ambientalmente correta.

A variável observável CS5 (Estou consciente que o consumo de produtos sustentáveis (verdes) é importante para o meio ambiente e a manutenção das gerações futuras) também apresentou uma alta carga fatorial $(0,797)$, o que está de acordo com a pesquisa de Severo, Guimarães e Dorion (2018), pois o consumo sustentável é aquele que visa a consumir adequadamente os recursos naturais, respeitando o meio ambiente para que as gerações futuras tenham as suas necessidades atendidas.

Neste cenário, a Tabela 3 apresenta a análise fatorial confirmatória para o Bloco 2 - MKT, o qual apresenta valores aceitáveis à validação da escala, pois a comunalidade é satisfatória, assim como as cargas fatoriais das variáveis observáveis. Na composição do Bloco 2, a variável observável que apresentou a maior carga fatorial $(0,816)$ foi a MKT1 (A propaganda tem uma grande influência na escolha dos produtos sustentáveis), demonstrando que os respondentes acreditam que a propaganda sustentável influencia na escolha de produtos, o que está de acordo com as premissas de Lee, Andrade e Palmer (2013) e Hoffmann et al. (2019), pois os consumidores estão sujeitos a estímulos de marketing, os quais apresentam-se como indutores de emoções sob a forma de propagandas, embalagens de produtos e posicionamento de marcas.

A variável observável MKT5 (Considero importante a divulgação do consumo consciente dos produtos verdes), contudo, também exibiu uma alta carga fatorial $(0,802)$, tornando primordial a divulgação do consumo consciente, pois, de acordo com Diamantopoulos et al. (2003), pode influenciar na decisão de compra, e isso tem gerado uma grande expansão de produtos verdes em diversos países.

Tabela 3 - Análise fatorial confirmatória para o Marketing Verde

\begin{tabular}{|c|c|c|c|}
\hline Bloco & Variáveis observáveis & Comunalidade & $\begin{array}{c}\text { Cargas } \\
\text { Fatoriais }\end{array}$ \\
\hline \multirow{5}{*}{$\begin{array}{l}\text { Marketing } \\
\text { Verde (MKT) }\end{array}$} & $\begin{array}{l}\text { MKT1 - A propaganda tem uma grande influên- } \\
\text { cia na escolha dos produtos sustentáveis. }\end{array}$ & 0,666 & 0,816 \\
\hline & $\begin{array}{l}\text { MKT2 - A propaganda sobre produtos sustentá- } \\
\text { veis me levou a realizar uma compra de produto } \\
\text { verde }\end{array}$ & 0,524 & 0,724 \\
\hline & $\begin{array}{l}\text { MKT3 - Se as lojas, onde habitualmente compro, } \\
\text { oferecerem produtos verdes eu substituiria a } \\
\text { compra de produtos convencionais por produtos } \\
\text { verdes. }\end{array}$ & 0,551 & 0,742 \\
\hline & $\begin{array}{l}\text { MKT4 - Considero importante uma melhor di- } \\
\text { vulgação sobre o descarte correto de resíduos } \\
\text { eletrônicos (pilhas, baterias, celulares, TV etc.) }\end{array}$ & 0,578 & 0,760 \\
\hline & $\begin{array}{l}\text { MKT5 - Considero importante a divulgação do } \\
\text { consumo consciente dos produtos verdes. }\end{array}$ & 0,643 & 0,802 \\
\hline
\end{tabular}

Fonte: Dados da pesquisa (2018). 


\section{Regressão linear múltipla}

Inicialmente realizou-se a análise da matriz de Correlação de Pearson, para verificar a multicolinearidade antes do processo da regressão linear múltipla, a qual permite descobrir se algumas variáveis independentes estão altamente correlacionadas, o que evita a multicolinearidade, posto que esta ocorre quando as correlações entre as variáveis encontram-se acima de 0,8 (WOOLDRIGGE, 2006). Consoante isso, a Correlação de Pearson apresentou baixas correlações entre as variáveis independentes, não ocorrendo a multicolinearidade entre as variáveis observáveis.

A regressão linear múltipla é uma análise estatística, descritiva e de inferência entre uma variável dependente $(\mathrm{Y})$ como efeito de múltiplas variáveis independentes (X) de causa (HAIR JR. et al., 2013). Conforme estes autores, a análise indica os efeitos cumulativos de um grupo de variáveis independentes (X1, X2, Xn) em uma variável dependente $(Y)$, da mesma forma que destaca os efeitos das variáveis independentes ou exploratórias $(Y=\beta 1 X 1+\beta 2 X 2+\beta 3 X 3+\ldots+\beta 0)$.

A pesquisa verificou a relação entre os construtos CS e MKT. O estudo analisa $O$ Modelo 1, tendo as médias do Construto CS (CS1, CS2, CS3, CS4 e CS5) como uma variável dependente (efeito) e MKT (MKT1, MKT2, MKT3, MKT4 e MKT5) como variável independente (Tabela 4).

Tabela 4 - Regressão linear múltipla

\begin{tabular}{|l|c|c|c|c|}
\hline Modelo & $\mathbf{R}$ & $\begin{array}{c}\mathbf{R} \\
\text { quadrado }\end{array}$ & $\begin{array}{c}\text { R quadrado } \\
\text { ajustado }\end{array}$ & $\begin{array}{c}\text { Erro padrão } \\
\text { da estimativa }\end{array}$ \\
\hline 1 & $0,661^{\mathrm{a}}$ & 0,437 & 0,434 & 0,61050 \\
\hline a. Preditores: (Constante), MKT5, MKT2, MKT3, MKT4, MKT1 \\
\hline b. Variável dependente: MenCS \\
\hline
\end{tabular}

Fonte: Dados da pesquisa (2018).

Os resultados da regressão linear múltipla apresentam um índice de explicação de $43,70 \%\left(R^{2}\right)$. Perante o exposto, a relação das variáveis do Marketing Verde (MKT), como o Consumo Sustentável (CS), apresenta uma intensidade moderada. Neste sentido, o MKT tem o propósito de transmitir ao consumidor as ações sociais, as práticas ambientais e o investimento da organização para melhoria do meio ambiente (NYILASY; GANGADHARBATLA; PALADINO, 2014; PAPADAS; AVLONITIS; CARRIGAN, 2017).

Vale também destacar que os achados da pesquisa retratam a importância de o MKT estar inserido na propagandas e mídias de produtos e serviços, pois pode sensibilizar um grande número de consumidores, aumentando o nicho de consumidores verdes, ou seja, os que estão cientes da problemática ambiental e da necessidade de ações humanas para a preservação dos recursos naturais, melhoria da qualidade de vida e o futuro das gerações (CHEN; CHANG, 2013; DELLARMELIN et al., 2018; GUILLEN-ROYO, 2019; DHANDRA, 2019).

\section{CONSIDERAÇÕES FINAIS}

Esta pesquisa analisou a influência do marketing verde no consumo sustentável, com o objetivo de mensurar as percepções de 820 consumidores do RN, uma vez que essas preferências, na sua maioria, estão pautadas pela influência das propagandas no momento das compras, as quais reverberam na mudança de hábitos e troca de produtos. 
Nesse contexto, Ottman e Paro (1994) e Chen e Chang (2013) destacam que o marketing verde surge da demanda de novas táticas, a qual produz abordagens de desafios-chave no que se refere ao verde. Desse modo, a produção de produtos e serviços verdes ampara-se por meio de impactos no desempenho de novas iniciativas, o que transmite aos consumidores credibilidade e apreciação pelo produto.

Os resultados da pesquisa evidenciam uma intensidade moderada $(43,7 \%)$ do marketing verde no consumo sustentável no RN. Estes achados denotam que o marketing verde contribui para comportamentos sustentáveis, pois evidencia-se que os consumidores percebem a problemática ambiental e visam um consumo consciente, o que corrobora os pressupostos de Ladhari (2007), posto que ocorre impacto das emoções sobre a satisfação, podendo ser inerente a diferentes percepções de satisfação, confiança e compromisso (SCHOEFER; DIAMANTOPOULOS, 2009), de forma que as emoções geram impacto positivo no consumo sustentável (SHETH; MITTAL; NEWMAN, 2001; DAS; VARSHNEYA, 2017; DONG et al., 2018). Não se deve esgotar, entretanto, os esforços do governo, das empresas, das instituições de ensino e da sociedade para a divulgação da problemática ambiental que se encontra generalizada, cabendo a todos os cidadãos a consciência de preservação dos recursos naturais, a diminuição do impacto ambiental e a atitude-comportamento, visando a um consumo sustentável.

As contribuições gerenciais do estudo estão atreladas às decisões gerenciais para o poder de compra versus as empresas, bem como a imagem verde das organizações pode contribuir para o desempenho organizacional, uma vez que as práticas de responsabilidade ambiental refletem positivamente junto aos consumidores, podendo alcançar os diversos stakeholders, que podem pautar seus investimentos a partir da postura ambiental da empresa. No que se refere às contribuições acadêmicas, o estudo validou estatisticamente os construtos da pesquisa, os quais poderão ser utilizados em outros estudos científicos.

Para estudos futuros, sugere-se uma análise em outras Regiões e Estados, bem como pesquisas qualitativas para entendimentos relacionados à motivação dos consumidores verdes, aprimorando, assim, os conhecimentos com inovações sustentáveis atreladas a uma futura compra sustentável. Diante do exposto, espera-se que os consumidores estejam dispostos a consumir produtos verdes, assim como as empresas a modificar seu segmento corporativo em busca de equilíbrio no retorno financeiro, na tentativa de suprir o que é socialmente desejável em termo ecológico.

Pode-se concluir que há uma crescente conscientização por parte dos consumidores verdes, entretanto é necessária maior divulgação dos produtos verdes, ou seja, o marketing verde pode adentrar novos mercados e consumidores com o objetivo de divulgar e informar a importância de um consumo sustentável. Divulgar também a problemática ambiental que vem impactando o planeta e a necessidade de mudanças de hábitos de consumo, o que pode ocasionar alterações nos processos e produtos organizacionais, podendo proporcionar vantagem competitiva perante a diferenciação de produtos/serviços verdes em relação às empresas concorrentes. 


\section{REFERÊNCIAS}

ALVES, R. R. Marketing ambiental: sustentabilidade empresarial e mercado verde. 1. ed. São Paulo: Editora Manole, 2016.

BEYLOT, A.; SECCHI, M.; CERUTTI, A.; MERCIAI, S.; SCHMIDT, J.; SALA, S. Assessing the environmental impacts of EU consumption at macro-scale. Journal of Cleaner Production, v. 216, p. 382-393, 2019.

BRAGA JUNIOR, S. S.; DA SILVA, D.; DE MORAES, N. R.; GARCIA, S. F. A. Verdadeiro ou falso: a percepção do consumidor para o consumo verde no varejo. Revista Brasileira de Marketing, v. 15, n. 3, p. 390-400, 2016. BYRNE, B. M. Structural equation modeling with AMOS: basic concepts, applications and programming. 2. ed. New York: Taylor \& Francis Group, 2010.

CHANG, K. C.; HSU, C. L.; HSU, Y. T.; CHEN, M. C. How green marketing, perceived motives and incentives influence behavioral intentions. Journal of Retailing and Consumer Services, 49, p. 336-345, 2019.

CHEN, Y. S.; CHANG, C. H. Greenwash and green trust: the mediation effects of green consumer confusion and green perceived risk. Journal of Business Ethics, v. 114, n. 3, p. 489-500, 2013.

CHEN, C. Y.; MATHUR, P.; MAHESWARAN, D. The effects of country-related affect on product evaluations. Journal of Consumer Research, v. 41, n. 4, p. 1.033-1.046, 2014.

DAHL, R. Green washing: do you know what you're buying? Environmental Health Perspectives, v. 118, n. 6, p. A246, 2010.

DHANDRA, T. K. Achieving triple dividend through mindfulness: More sustainable consumption, less unsustainable consumption and more life satisfaction. Ecological Economics, v. 161, p. 83-90, 2019.

DANGELICO, R. M.; VOCALELLI, D. “Green Marketing”: an analysis of definitions, strategy steps, and tools through a systematic review of the literature. Journal of Cleaner Production, v. 165, p. 1.263-1.279, 2017. DAS, G.; VARSHNEYA, G. Consumer emotions: Determinants and outcomes in a shopping mall. Journal of Retailing and Consumer Services, v. 38, p. 177-185, 2017.

DELLARMELIN, M. L.; MORAES, J. P.; HOECKESFELD, L.; SEVERO, E. A.; HAHN, I. S. The influence of green branding on purchase intention, willingness to pay and consumers' emotional response: an experimental study. Latin American Journal of Management for Sustainable Development, v. 4, n. 1, p. 69-82, 2018.

DE OLIVEIRA, V. M.; CORREIA, S. É. N.; GOMEZ, C. R. P. Cultura de consumo, sustentabilidade e práticas empresariais: como as empresas podem contribuir para promover o valor simbólico da sustentabilidade nas atividades de consumo? Revista de Gestão Ambiental e Sustentabilidade-GeAS, v. 5, n. 1, p. 61-77, 2016.

DIAS, R. Marketing ambiental-ética, responsabilidade social e competitividade nos negócios. 1. ed. São Paulo: Editora Atlas, 2008.

DIAMANTOPOULOS, A.; SCHLEGELMICH, B.; SINKOVICS, R.; BOHLEN, G. Can socio-demographics still play a role in profiling green consumers? Journal of Business Research, v. 56, n. 6, p. 465-480, 2003.

DONG, X.; LI, H.; LIU, S.; CAI, C.; FAN, X. How does material possession love influence sustainable consumption behavior towards the durable products? Journal of Cleaner Production, v. 198, p. 389-400, 2018. D'SOUZA, C.; TAGHIAN, M.; LAMB, P. An empirical study on the influence of environmental labels on consumers. Corporate Communications: An International Journal, v. 11, n. 2, p. 162-173, 2006.

ENGEL, J. F.; BLACKWELL, R. D.; MINIARD, P. W. Comportamento do consumidor. 8. ed. Rio de Janeiro: LTC, 2000.

FRANCO, M. P. V. Uma questão de poder: consumo sustentável e suficiência ecológica como discursos alternativos em prol da sustentabilidade. Sociedade e Natureza, v. 31, p. 1-21, 2016.

GENG, D.; LIU, J.; ZHU, Q. Motivating sustainable consumption among chinese adolescents: An empirical examination. Journal of Cleaner Production, v. 141, p. 315-322, 2017.

GIL, A. C. Métodos e técnicas de pesquisa social. 5. ed. São Paulo: Atlas, 2006.

GOMES, D. V. Educação para o consumo ético e sustentável. Revista Eletrônica do Mestrado em Educação Ambiental, v. 16, p. 1-14, 2006.

GUILLEN-ROYO, M. Sustainable consumption and wellbeing: does on-line shopping matter? Journal of Cleaner Production, v. 229, p. 1.112-1.124, 2019.

GUIMARÃES, J. C. F.; SEVERO, E. A.; DORION, E. C. H.; COALLIER, F.; OLEA, P. M. The use of organizational resources for product innovation and organizational performance: A survey of the brazilian furniture industry. International Journal of Production Economics, v. 180, p. 135-147, 2016.

HAIR JR., J. F.; BLACK, W. C.; BARDIN, B. J.; ANDERSON, R. E. Multivariate data analysis. 7. ed. New York: Pearson Education Limited, 2013.

HOFFMANN, S.; MAI, R.; LASAROV, W.; KRAUSE, J. S.; SCHMIDT, U. Hungry bellies have no ears. How and why hunger inhibits sustainable consumption. Ecological Economics, v. 160, p. 96-104, 2019.

IBGE. Instituto Brasileiro de Geografia e Estatística. Perfil regional. Disponível em: www.ibge.org.br. Acesso em: 10 de nov. 2018.

KOTLER, P.; KELLER, K. L. Administração de marketing. São Paulo: Pearson Education, 2012. 
LADHARI, R. The effect of consumption emotions on satisfaction and word-of-mouth communications. Psychology \& Marketing, v. 24, n. 12, p. 1.085-1.108, 2007.

LEE, C. J.; ANDRADE, E. B.; PALMER, S. E. Interpersonal relationships and preferences for mood-congruency in aesthetic experiences. Journal of Consumer Research, v. 40, n. 2, p. 382-391, 2013.

LEE, N.; HOOLEY, G. The evolution of "classical mythology" within mar eting measure development. European Journal of Marketing, v. 39, p. 365-385, 2005.

LIM, W. M. Inside the sustainable consumption theoretical toolbox: Critical concepts for sustainability, consumption, and marketing. Journal of Business Research, v. 78, p. 69-80, 2017.

LIRA, F. T. Fatores que influenciam a valorização de produtos ecológicos por consumidores ecologicamente conscientes. Revista de Gestão Social e Ambiental, v. 12, n. 2, p. 90-107, 2018.

MALHOTRA, N. K. Pesquisa de marketing: uma orientação aplicada. Porto Alegre: Bookman Editora, 2012. MARCONI, M. A.; LAKATOS, E. M. Técnicas de pesquisa. 8. ed. São Paulo: Atlas, 2017.

MARDIA, K. V. The effect of nonnormality on some multivariate tests and robustness to nonnormality in the linear model'. Biometrika, v. 58, n. 1, p. 105-121, 1971.

NYILASY, G.; GANGADHARBATLA, H.; PALADINO, A. Perceived greenwashing: The interactive effects of green advertising and corporate environmental performance on consumer reactions. Journal of Business Ethics, v. 125, n. 4, p. 693-707, 2014.

OLIVEIRA, V. M.; GOMEZ, C. R. P.; CORREIA, S. É. N. Papéis das empresas e o consumo sustentável na visão de especialistas brasileiros. Revista de Gestão Social e Ambiental, v. 12, n. 2, p. 55-70, 2018.

OTTMAN, J. A. Green marketing. [S.I.]: NTC Publishing Group, 1993.

OTTMAN, J. A.; PARO, M. N. Marketing verde: desafios e oportunidades para a nova era do marketing. São Paulo: Makron Books, 1994.

PAAVOLA, J. Economics, ethics and green consumerism. In: Exploring Sustainable Consumption, p. 79-94, 2001. PAPADAS, K-K.; AVLONITIS, G. J.; CARRIGAN, M. Green marketing orientation: Conceptualization, scale development and validation. Journal of Business Research, v. 80, p. 236-246, 2017.

PRAHALAD, C. K.; HART, S. L. Raising the bottom of the pyramid: Strategies for sustainable growth. Ann Arbor, v. 1.001, p. 48.109, 2000.

PORTILHO, F. Consumo sustentável: limites e possibilidades de ambientalização e politização das práticas de consumo. Cadernos Ebape, v. 3, n. 3, p. 1-12, 2005.

RYAN, K.; DANYLCHUK, A.; JORDAAN, A. Consideration of scales in offshore wind environmental impact assessments. Environmental Impact Assessment Review, v. 75, p. 59-66, 2019.

SCHOEFER, K.; DIAMANTOPOULOS, A. A typology of consumers' emotional response styles during service recovery encounters. British Journal of Management, v. 20, n. 3, p. 292-308, 2009.

SEVERO, E. A.; DE GUIMARÃES, J. C. F.; DORION, E. C. H. Cleaner production, social responsibility and eco-innovation: Generations' perception for a sustainable future. Journal of Cleaner Production, v. 186, p. 91-103, 2018.

SEVERO, E. A.; GUIMARÃES, J. C. F.; BRITO, L. M. P.; DELLARMELIN, L. M. Environmental sustainability and sustainable consumption: the perception of baby boomers, generation $\mathrm{x}$ and $\mathrm{y}$ in brazil. Revista de Gestão Social e Ambiental, v. 11, n. 3, p. 92-111, 2017.

SHARMA, R.; JHA, M. Values influencing sustainable consumption behaviour: Exploring the contextual relationship. Journal of Business Research, v. 76, p. 77-88, 2017.

SHETH, J. N.; MITTAL, B.; NEWMAN, B. I. Comportamento do cliente. São Paulo: Atlas, 2001.

STRAUGHAN, R. D.; ROBERTS, J. A. Environmental segmentation alternatives: a look at green consumer behavior in the new millennium. Journal of Consumer Marketing, v. 16, n. 6, p. 558-575, 1999.

TIWARI, S.; TRIPATHI, D. M.; SRIVASTAVA, U.; YADAV, P. K. Green marketing-emerging dimensions. Journal of Business Excellence, v. 2, n. 1, p. 18-23, 2011.

WALKER, R. H.; HANSON, D. J. Green marketing and green places: A taxonomy for the destination marketer. Journal of Marketing Management, v. 14, n. 6, p. 623-639, 1998.

WOOLDRIGGE, J. M. Introdução à econometria: uma abordagem moderna. São Paulo: Pioneira Thompson Learning, 2006.

XU, S.; CHU, C.; ZHANG, Y.; YE, D.; WANG, Y.; JU, M. Entangled stakeholder roles and perceptions of sustainable consumption: An evaluation of sustainable consumption practices in Tianjin, China. Journal of Environmental Management, v. 223, p. 841-848, 2018.

ZHANG, L.; LI, D.; CAO, C.; HUANG, S. The influence of greenwashing perception on green purchasing intentions: The mediating role of green word-of-mouth and moderating role of green concern. Journal of Cleaner Production, v. 187, p. 740-750, 2018.

ZINKHAN, G.; CARLSON, L. Green advertising and the reluctant consumer. Journal of Advertising, v. 24, n. 2, p. 1-6, 1995. 